\title{
Educar para ser hoy. Una perspectiva de educación diferente y creativa
}

\section{Concepció Torres Sabatéa}

Rebut: 21/11/2011 Acceptat: 01/12/2011

\section{Resum}

En este artículo se presenta la realidad de la escuela y sus contrastes educativos desde una perspectiva de escuela libre, flexible, abierta... y basandose en algunos proyectos que actualmente están iniciándose en diversos lugares del mundo con una perspectiva histórica que argumenta y apoya este modelo.

Cuestiona algunos aspectos pedagógicos que han quedado olvidados en nuestras aulas, pero que algunos centros han recuperado gracias a las iniciativas de profesorado y familias.

Palabras clave: educación, creatividad, escuela, cambios, sociedad y realidad.

\section{To educate to be today. A perspective of different and creative education}

\begin{abstract}
This article presents the reality of the school and its educational contrasts from a free school, flexible, open ... and based on some starting projects currently in various places in the world with a historical perspective that argues and supports this model. Pedagogical questions some aspects have been forgotten in our classrooms, but some centers have recovered thanks to the efforts of teachers and families.
\end{abstract}

Key words: education, creativity, school change, society and reality.

\section{0.- Introducción}

"Por lo general creemos que el mejor camino para conseguir personas completas consiste en adaptar al niño a los ideales del hombre maduro. (...) La madurez y la cultura no son de ningún modo una creación del adulto o del sabio, sino más bien del niño y del salvaje. Dejemos que

\footnotetext{
${ }^{\text {a }}$ Departament de Pedagogia - Universitat Rovira i Virgili
} 
crezcan niños completos; para ello olvidemos tanto como podamos que un día serán hombres y mujeres. Eduquemos a los niños a ser niños y no siguiendo el ideal del adulto modélico sino medidas infantiles. El mejor hombre no es nunca aquel que fue menos niño, sino, al contrario; es aquel que cuando llega al trigésimo año de su vida encuentra reunido en su corazón el maravilloso tesoro de la infancia." (Ortega y Gasset, 1921)

\section{1.- ¿Es posible una escuela diferente?}

Se presenta a la escuela como un lugar de encuentro y de aprendizaje formando parte de un sistema que regula desde las profesiones a las conductas individuales y colectivas para perpetuar el engranaje de la propia sociedad, cada uno en su sitio como nos indica J.M. Mendiluce en el siguiente texto:

\footnotetext{
"Nos van robando la ciudadanía a base de "zapatero a tus zapatos", que dejaría más y más la cosa pública en manos de los profesionales de la política. Médicos a curar, abogados a defender, escritores a escribir, agricultores a la subvención, obreros... al paro. Así, cada cual a lo suyo o a lo que les dejan en el reparto social" (J. M. Mendiluce, 1998, 19).
}

No hay que perpetuar sino evolucionar. Somos conscientes que la escuela ya no es el único lugar, ni de encuentro ni de aprendizaje, por ello debe competir con esos otros espacios.

Hoy a la escuela se le dan más responsabilidades de las que puede asumir y se le delegan fracasos sociales y sus problemáticas, para que las resuelva. La política social debe recuperar sus propias responsabilidades para asumirlas y no derivarlas a la escuela. La escuela refleja los propios conflictos sociales, pero estos no se crean dentro de ella, sino fuera, aunque en demasiadas ocasiones estallan dentro. De esta forma se podrán prevenir y corregir los desajustes sociales y educativos.

Ante esta realidad debemos buscar alternativas educativas que mejoren el desarrollo personal de los alumnos/as.

Se intuye la necesidad de utopías que debemos buscar y crear para llegar a la verdadera transformación social, y puede conseguirse a través de la educación y con la escuela como uno de los elementos claves coordinados con otros ámbitos educativos que actualmente hacen funciones educadoras.

Las culturas debe presentarse dentro de un sistema educativo como un elemento cohesionador y de identidad de un colectivo, no distorsionador. Para ello es necesario tener en cuenta en nuestra realidad social la diversidad cultural, y analizar los diversos planteamientos para abordar estos conceptos y realidades, en la escuela desde una perspectiva global y enriquecedora para todos los colectivos a favor de la convivencia dentro de una sociedad cada vez más multicultural. Sin sentirse identificado (identidad) el aprendizaje se desarrollará de manera más compleja y generará conflictos en las relaciones personales. El contexto educativo debe considerar todos los contextos culturales (y la sociedad también). 
Un factor importante para la mejora educativa es la inversión económica que se destine a ella.

El concepto de "economía" está presente de manera permanente en nuestra sociedad por ser imprescindible. En el ámbito educativo, este concepto, debe entenderse como "inversión" aunque siempre acaba siendo insuficiente. Muchas de las escuelas alternativas que hoy existen han superado su "crisis económica" para mantener la escuela creando soluciones alternativas a la dependencia del Estado, incluso desarrollando proyectos comerciales que siguen su filosofía de respeto al entorno, etc.

La relación escuela - nación (a la que relaciono con política) - industria (a la que relaciono con economía) actualmente es compleja pero existente y está conectada directamente con la cuestión de organización y distribución del conocimiento según las necesidades sociales.

Es necesario tener unos gestores económicos que sepa reorganizar el presupuesto educativo.

El hecho de que el Estado invierta en educación no debe ser a cambio de que todos los centros educativos deban organizarse de una misma manera.

El Estado es responsable de la educación pero a la vez debe ofrecer más posibilidades de experimentación ante la escuela plural, flexible e intercultural que hoy forma parte de nuestra sociedad.

La escuela debe tener la libertad de desarrollar proyectos que permitan el desarrollo del niño/a, que investigue y sea capaz de crear el espacio y tiempo del niño/a, generando si es necesario, una utopía que ayude a reconocer el camino con la dirección a seguir.

\section{2.- La historia.}

En el desarrollo del siguiente punto podremos observar propuestas de diversos autores que han experimentado con el proyecto de una escuela plural, flexible, intercultural que trabaja para el verdadero desarrollo del niño/a, que retoma el centro educativo como centro de investigación y que crea el tiempo y el espacio del niño/a creyendo en la utopía como elemento para marcar una dirección a seguir.

La historia de la educación nos ha ofrecido ya diversos modelos de escuela que remarcan la importancia del niño/a como centro del proceso de enseñanzaaprendizaje utilizando metodologías activas y participativas.

Algunos de los modelos que podemos considerar más relevantes han sido:

- El movimiento de la Escuela Nueva de finales del S. XIX y principios del XX, con sus grandes representantes: John Dewey, Claparède, Ferrière, Maria 
Montesori, Decroly, todos ellos organizaban sus metodologías y materiales para un mejor desarrollo del niño/a. Su máxima era el respeto a la infancia.

Toda la filosofía de este movimiento se basó en el naturalismo pedagógico de Rousseau, Vives y Montaigne (crecimiento natural).

- La teoría liberadora de Paolo Freire, esa pedagogía crítica que debe plantearse a los futuros maestros/as y pedagogos/as. A la vez que nos retoma el concepto de utopía como una necesidad para el desarrollo personal y profesional e incluso para la transformación.

- La concreción de nuestro sistema educativo en las últimas leyes ( desde laLOGSE a la LOE), que desarrollan la aplicación del mismo, destaca la necesidad de la individualidad del alumno, la inclusión (escuela inclusiva....). Nos hacen recordar las experiencias educativas de Don Lorenzo Milani y E. Mounier donde la aceptación del otro, el respeto, la autenticidad, etc. eran aspectos a conseguir en la educación personalista, teniendo en cuenta al alumno/a como individuo y como ciudadano que forma parte de un colectivo (sociedad), la llamada teoría personalista, que suma la sociológica y la individualista. Pero realmente vemos estos aspectos en nuestras aulas?.

- La teoría libertaria que nos recuerda la aportación de Alexander Sutherland Nelly con su escuela Summerhill, que busca la felicidad del niño/a creando espacios i materiales para favorecer el desarrollo personal y madurativo del alumno.

- La teoría contra la institución escolar con:

- Paul Goodman que critica a la escuela por considerarla una "trampa universal que no sirve para nada" y a la educación obligatoria como "deseducación". Propone que la educación tenga objetivos más humanos, operativos, que trabajen la creatividad y la participación.

- Everett Reimer ve a la escuela como un instrumento de control que garantiza la persistencia de un modelo social concreto.

- John Holt presenta la libertad como elemento clave de la función de la escuela, se debe dejar que el alumno planifique su propio aprendizaje de manera libre e independiente.

- Las directrices generales de la educación deben ser además de la libertad, el respeto al medioambiente, saber compaginar trabajo y ocio, la paz y la lucha contra el racismo.

- Ivan Illich, y E. Reimer concretan que la escuela es la culpable del control social y plantea como una de las tareas del maestro la investigación para abrir nuevos caminos hacia la mejora de las funciones de la escuela. 
Debemos retomar estas filosofías y actuar para su proyección.

\section{3.- La realidad.}

La suma de muchas de estas experiencias han favorecido la creación de escuelas alternativas a la educación convencional con el objetivo de respetar el ritmo de aprendizaje del niño/a. Escuelas que han apostado por una pedagogía abierta, activa y creativa.

Actualmente, en muchos países, existen alternativas a la educación estatal. En EEUU las escuelas libres y la educación en casa (homeschooling) no es un fenómeno raro como en España. La escuela Sudbury Valley es un referente mundial desde su creación en 1968 y modelo para muchas de estas escuelas.

En nuestros países vecinos (Portugal, Italia y Francia) tampoco es excepcional este tipo de educación. En Inglaterra funciona desde 1921 la escuela libre más conocida del mundo: Summerhill, y es posible acogerse a la exención del Currículo Nacional. En Ecuador, El Pesta (Pestalotzzi desde 1977 hasta 2005 que se transforma en un CEPACentro para actividades autónomas) ha sido el desencadenante de muchas escuelas libres que están apareciendo en España y desde hace ya algunos años en países del norte de Europa.

En muchos países, la educación en casa es legal, lo cual proporciona cobertura a quienes crean espacios educativos de este tipo; otros países, como Dinamarca, consiguen sin dificultades la legalización y financiación estatal de escuelas no convencionales. En España a pesar de no estar contemplado en la ley, existen iniciativas de este tipo que funcionan desde hace ya algunos años. La escuela Paideia en Mérida desde mediados de los años 70, al igual que O' Pelouro en Galicia, la escuela libre Micael desde 1979 en Las Rozas (Madrid), Donyets en Valencia y el Roure en Mediona (Barcelona) desde hace unos 13 años, etc.

Algunos ejemplos de iniciativas alternativas de más reciente creación son: La Violeta en Madrid, Alavida en Valdemorillo (Madrid), Ojo de Agua en Alicante, Pequeña Rebeldía en Murcia, Madreselva en Cáceres, Escola Lliure Hort del Pi en Valencia, La Caseta, La Mangarrufa, Moixana en Barcelona, La Salara, El sorral, El Submarí Lila en Tarragona, etc.

También hay que reconocer que dentro de la educación pública existen algunas iniciativas interesantes de maestros preocupados que intentan mejorar el propio contexto escolar hacia una educación más respetuosa con el entorno vital del niño/a y adolescente. Por ejemplo el colegio Riera de Ribes en Sant Pere de Ribes (Barcelona) y el proyecto Atlántida. Y en escuelas privadas la mayoría ya concertadas como Escola Lys, Nusica, Escola Els Arcs (esta de secundaria), tienen también proyectos alternativos dentro de la legalidad.

Un artículo de El País del día 13 de octubre de 2003 presenta los siguientes datos: 


\begin{abstract}
"Más de 4.000 alumnos estudian en seis colegios públicos y privados reconocidos oficialmente pero que enseñan de manera diferente: no imparten asignaturas como tales, ni hacen exámenes. Ponen en marcha de forma generalizada experiencias que otros centros realizan aisladamente" (Elena Sevillano, 2003, 30).
\end{abstract}

Todas estas iniciativas abordan los intereses de la infancia a través del respeto a su libertad, su tiempo, a la participación, a la autonomía, a la identidad, a la interacción constante con el entorno, a la globalidad de los conocimientos que se presentan como deseo, a la investigación, etc. Se van creando ambientes relajados donde la función del adulto (padres, educadores....) es estar presentes para favorecer la relajación del niño/a que se da cuenta que esta acompañado, pero sin intervención directa del propio adulto si primero no se le pide la ayuda.

Se realizan ofertas o diversidad de propuestas de aprendizaje para que sean elegidas libremente. Clases o espacios abiertos a personas y a conocimientos.

Su objetivo principal es la realización del potencial humano en todas sus etapas de desarrollo respetando los procesos de vida del niño/a siempre: en su primera etapa sensoriomotriz, en las etapas siguientes sumando el desarrollo de los sentidos, hasta la etapa operativa donde todas las actividades y juegos son utilizados para obtener reglas y aplicarlas en contextos diferentes, han asumido la comprensión simbólica y hablan de lo que están haciendo (comprender - experimentar - entender).

El niño/a quiere descubrir el mundo a través de su propia actividad y de sus propios intentos, ante estos intentos el adulto juega un papel muy importante, ya que su cercanía a través de un abrazo, de una caricia, de un consuelo, de una palabra, etc. les da seguridad (lo emocional debe estar cubierto para poder desarrollar el aprendizaje).

Remarcan que las necesidades internas del niño/a y del adolescente son el probar, sentir y percibir. No quieren anteponer a este desarrollo las expectativas de la sociedad ni plantarlas "para asegurar la continuidad del sistema" (Ángel García del Dujo; Fernando Gil Cantero, 2009 Ponencia).

A partir de los 14 años, ahora quizás antes, el adolescente ya se pregunta quién es y quiere descubrir su propio yo en el mundo; pero debe descubrirlo él mismo. Para esta etapa estas iniciativas alternativas a la escuela estatal, preparan también espacios y materiales para desarrollar las cualidades verdaderamente humanas en este ambiente. La utilización del lenguaje propio y extranjero es uno de los elementos de deseo para la comunicación con sus iguales y con los adultos y la autorreflexión. Se presenta la comunicación de manera natural como una necesidad porque en nuestro mundo moderno cada vez se dan menos ocasiones para las conversaciones personales, ni en casa ni en la escuela.

A todos los niveles también se determinan los "límites" como una necesidad, no para el control del individuo, sino para conseguir la convivencia y dar seguridad. Estos 
límites ayudan a reducir vivencias inadecuadas que pueden favorecer en el niño/a el crear mecanismos de defensa, con ello se ayuda a socializar las vivencias.

Demasiadas veces el pensamiento lógico ha substituido a los sentimientos en nuestros sistemas educativos, modificando esta característica ya avanzaríamos hacia una educación con sentido. Pensando que el aprendizaje debe estar dirigido desde el interior (el propio individuo) ya que el que proviene del exterior (siempre impuesto por el mundo de los adultos) va en contra de la naturaleza y tarde o temprano ocasiona conflictos.

Toffler (1983), ya da algunas ideas de cómo mejorar nuestros sistemas educativos destacando la posibilidad de una organización móvil (abandonar la rigidez del sistema); descentralización; introducción de los escolares en campos de la vida y del trabajo existentes en la sociedad actual; flexibilización de la rigidez de los horarios de asignaturas (se aprende globalmente) y de las divisiones por clases. Se deberían crear "planes de estudios elásticos".

Sobre algunos de estos aspectos Rebeca Wild en su obra "Educar para ser" nos expone su crítica a nuestro sistema educativo:

\begin{abstract}
"en nuestros planes de estudio existe una laguna especialmente dolorosa por lo que respecta al trato con los mundos internos y al intercambio dinámico entre interior y exterior, justo lo que hace de nuestra vida algo digno de vida. (...)

Si estás bien sentado y calladito, no intentas mirar por la ventana o hablar con tu compañero, si tienes las manos bien colocadas sobre la mesa y me escuchas, entonces podrás aprender algo con sentido. Después, se abren los libros de texto y el niño ve el mundo a través de los cristales de las gafas de una comisión de expertos pedagogos. El maestro explica una cosa, escribe en la pizarra, dirige conversaciones. Los niños escuchan, escriben lo que pone el maestro en la pizarra y, en el mejor de los casos, dan las respuestas "correctas" que se esperan de ellos." (Rebeca Wild, 2005, 158).
\end{abstract}

Para todos estos aspectos presentados hay que favorecer que nuestro sistema vaya acercándose al verdadero respeto de la infancia y la adolescencia, buscando el concretar cual es el lugar de ambas en nuestra sociedad y a la vez, a crear más espacios de seguridad para conseguir el crecimiento integral dentro de un proceso natural de desarrollo, creando las condiciones adecuadas para que este proceso se realice sin esperar que ni la política, ni otras estructuras sociales lo resuelvan. Desde la formación del profesorado (entendiéndola ya a lo largo de la vida) podemos preparar ambientes para potenciar conscientemente el aprendizaje y la investigación, una formación que debe entenderse como un viaje personal para cambiar (Pilar Iranzo, 2009), sin olvidar nuestra realidad: una era "líquida" (Bauman, 2009), una "era del vacío" (Lipovetsky, 2002), que estamos "educando en una cultura del espectáculo" (Joan Ferrés, 2000). Una "Sociedad educadora" (J. Trilla) que identifica que recibimos tres tipos de educación que pueden ser incluso contrarias: la de nuestros padres, la de nuestros maestros y la del mundo. Hay que buscar el equilibrio entre los tres. 
Se abordan estos temas creando cosas como "ciudad educadora", "planes de entorno", "planes de inclusión", "escuelas que funcionan por proyectos"....pero no abordamos las cuestiones des de la raíz, debemos analizar las aplicaciones de las leyes que regulan el sistema educativo y a la vez atrevernos a provocar a los futuros maestros para que realmente sean críticos-constructivos (con propuestas concretas) con los sistemas que no funcionan o pueden mejorar.

En los documentos oficiales educativos, todo nuestro sistema educativo esta muy completo, reglado y detallado le queda desarrollar todo su potencial práctico.

Finalizando la reflexión redacto un diálogo del texto de (Octavi Fullat, 2006) proponiendo al lector el cambio entre la palabra "fotografía" por la de "sistema educativo" pensando que los políticos son los que participan de la creación del sistema educativo:

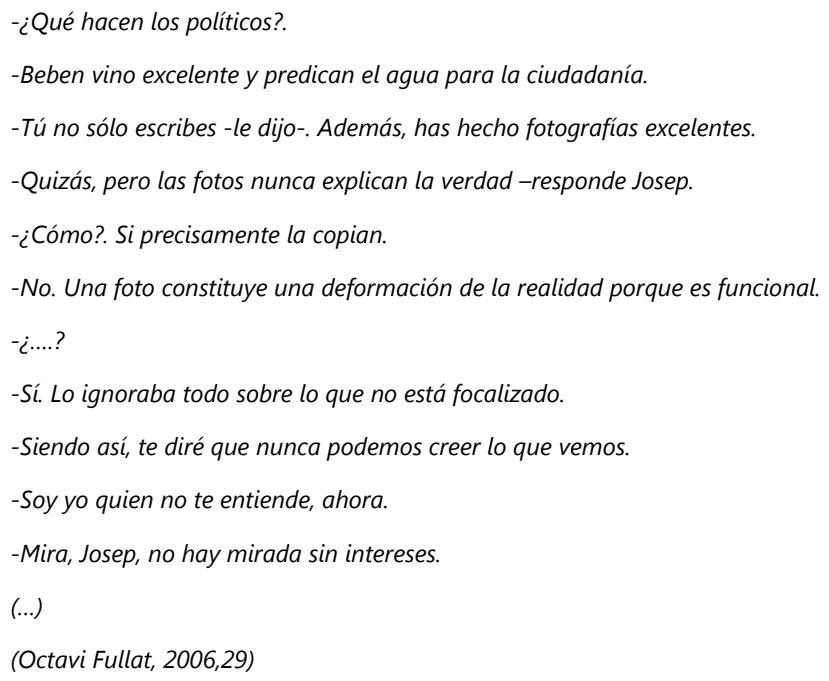

Podemos retomar las lecturas a favor de la infancia de Francesco Tonucci para comprender perfectamente que los niños/as también existen y necesitan sus espacios de juego, creación, aprendizaje y desarrollo. "El niño no es aquello que se ve desde arriba".

Busquemos como profesionales de la educación las iniciativas que ayuden a resolver y mejorar nuestro sistema educativo para mejorar los espacios del niño/a, sabiendo que uno de los más significativos es el espacio-escuela y que esta debe relacionarse con la familia, el entorno, el medio ambiente, las estructuras políticas, las estructuras pedagógicas, etc.

La respuesta a la cuestión expresada en la introducción (¿Es imprescindible tanta regulación concreta elaborada más con estructura política que educativa?), depende de cada uno de nosotros/as, pero estoy convencida que podemos conseguir el 
desarrollo de un sistema educativo más flexible y que responda más a las necesidades individuales de cada alumno.

Podemos educar para ser hoy.

\section{4.- Conclusiones: de la realidad a la creatividad.}

Para conseguir una buena escuela hay que incluir el desarrollo de la capacidad creativa, que no debe ser exclusiva de la expresión plástica, sino que debe ser una prioridad para preparar de manera crítica, activa y eficiente a los alumnos.

Para poder trabajar la creatividad, las escuelas comentadas en el punto anterior consideran necesario que el niño/a pueda moverse con libertad por los diferentes espacios educativos, tener espacios al aire libre para provocar un buen estado físico. Las aulas deben entenderse como espacios polivalentes donde se puedan realizar diversas actividades.

Otro aspecto importante es emocional, no se coartan sus propuestas, todo es importante. Hay que aprender a escuchar.

El hecho de que pueda aprender sobre sus propios intereses facilita la adquisición de conocimientos, que no pierda la curiosidad y a la vez un aprendizaje le lleva a otro ya que se plantean nuevos retos constantemente. Consiguen desarrollar sus propios proyectos de aprendizaje.

El actual modelo educativo debe superar el modelo que se inició con la revolución industrial para acercarlo a la era de la información. Hay que repensar el sistema y ampliar el grupo de personas que pueden intervenir en el proceso educativo, no sólo los maestros pueden ofrecer sus conocimientos, hay que invitar a toda la comunidad educativa para un planteamiento más global con la participación de agentes del entorno que pueden aportar conocimiento (padres, madres, abuelos/las, otros familiares, personas cercanas al barrio o al municipio, etc.).

Es necesario crear un modelo en el que los alumnos deben ser los que planteen los problemas y ellos mismos busquen las soluciones. Este papel activo favorece la participación, la motivación y al ver que los conocimientos dan respuesta a los propios intereses se mantiene la actitud positiva hacia el aprendizaje.

\section{Referencias}

Bauman, Z. (2009). El arte de la vida. De la vida como obra de arte. Barcelona, Paidós.

Ferres, J. (2000). Educar en una cultura del espectáculo. Barcelona. Paidós.

Fullat, O. (2006). La meva llibertat. Romanyà Valls. Angle editorial.

Iranzo, P. (2009). Innovando en educación. Formarse para cambiar un viaje personal. Vilafranca del Penedès. Erasmus ediciones. 
Lipovetsky, G. (2002). La era del vacío. Barcelona. Anagrama.

Martínez Bonafé, J. y otros (2003). Ciutadania, poder i educació. Barcelona. Editorial Graó.

Mendiluce, J.M. (1998). Tiempo de rebeldes ciudadanía y participación. Barcelona. Editorial Planeta.

Ortega y Gasset, J. (1921). Biología y pedagogía. En Obras completas. Madrid: AlianzaRevista Occidente 1983-1989.

Pendi Iyanga, A. (1998). La educación contemporánea. Teorías e instituciones. Valencia: Nau llibres.

Sevillano, E. (2003). Una forma distinta de aprender. El País, p.30. Madrid.

Toffler, A. (1990). El "shock" del futuro. Esplugues de LLobregat: Plaza \& Janés.

Tonucci, F. (2004). Quan Els infants diuen Prou!. Barcelona. Editorial Graó.

Wild, R. (2005). Educar Para ser. Vivencias de una escuela activa. Barcelona: Editorial Herder.

Wild, R. (2006). La vida en una escuela no directiva. Barcelona: Editorial Herder.

\section{Nota biográfica}

M. Concepció Torres Sabaté: Doctora en pedagogía. Profesora del Departamento de Pedagogía de la Facultad de Ciencias de la Educación y Psicología de la Universidad Rovira y Virgili. Impartiendo clases en Pedagogía, Magisterio y Educación Social.

Sus ámbitos de investigación son la Unión Europea y la educación con la Tesis "La Dimensión Europea de la Educación en los Centros d'ESO del Tarragonés", presentada en el 2004 y de la cual se han publicado diversos artículos: "La política universitaria y la Unión Europea", "La dimensión europea de la educación en los documentos básicos referenciales".

Y el voluntariado de las cuales se han generado diversas publicaciones: "Estudio del voluntariado en las universidades de Girona, Ramon Llull y Rovira i Virgili"; "Situación actual del voluntariado hospitalario en España"; "Educación, voluntariado y participación en la Unión Europea"; Inmigración y voluntariado" y "Pobreza y voluntariado".

Ha trabajo en la formación de jóvenes, adultos y padres; la educación en valores, en el tiempo libre, etc.

Ha colaborado en la publicación "Educación en valores, actitudes personales y ética profesional en el marco del espacio europeo de educación superior". 